\title{
Alles ist Verfassung
}

\section{Die 59. Assistententagung im Öffentlichen Recht in Frankfurt am Main (Wolfgang-von-Goethe- Universität) vom 19. bis 23. Februar 2019}

Sabrina Müller und Arno Schönberger"

\section{A. Einleitung}

Die diesjährige Assistententagung im Öffentlichen Recht in Frankfurt am Main als dem Ort, der vor allem wegen der dortigen Paulskirche zurecht als "Wiege der deutschen Demokratie" bezeichnet wird, stand unter dem Thema "Verfassungen Ihre Rolle im Wandel der Zeit" und beleuchtete die aktuellen dreifachen Verfassungsjubiläen von 1849 (Paulskirchenverfassung), 1919 (Weimarer Reichsverfassung) und 1949 (Grundgesetz). Dabei ging es nach der eigenen Beschreibung des Frankfurter Organisationsteams darum, die Herausforderungen zu betrachten, denen Verfassungen in verschiedenen Epochen immer wieder aufs Neue ausgesetzt sind. Besonderes Augenmerk wurde dabei auf ihre Widerstandsfähigkeit und Flexibilität im Angesicht gesellschaftlicher und technischer Veränderungen gelegt. Überdies bestand durch die spezifisch nationalverfassungsrechtlichen Beiträge der deutschsprachigen Kollegen aus Österreich und der Schweiz auch die wertvolle Gelegenheit zu verfassungsvergleichenden Studien.

Das Anliegen des vorliegenden Beitrags als Tagungsbericht besteht weniger darin, den genauen Verlauf der Tagung und ihres Programms sowie der entsprechend weitergeführten Diskussionen nachzuzeichnen als stattdessen vielmehr das allgemeine Stimmungsbild aufzufangen. Dabei bezieht sich der Begriff Stimmungsbild auf zwei Aspekte, namentlich einerseits auf das Tagungsthema und andererseits auf das in dessen Umsetzung zusammengestellte Tagungsprogramm. Dabei soll es schwerpunktmäßig auch darum gehen, das Thema der diesjährigen Tagung mit ihren inhaltlichen Programmpunkten in den allgemein laufenden Diskurs im Öffentlichen Recht einzuordnen.

\section{B. Stimmungsbild}

\section{Tagungsthema}

Verfassungen bilden die rechtliche Basis eines Gemeinwesens. Weil ein Gemeinwesen in seiner Entwicklung und in seinen Entscheidungen naturgemäß nie stehen bleibt, sondern einem ständigen Wandel unterworfen ist, befindet sich die Verfassung eines solchen in dem andauernden Dilemma, gleichermaßen eine gewisse Grundstabilität aufweisen und so den immer gleichbleibenden wesentlichen kon-

* Sabrina Müller ist wissenschaftliche Mitarbeiterin am Lehrstuhl für deutsches und ausländisches Öffentliches Recht, Staatskirchenrecht und Völkerrecht (Prof. Dr. von Ungern-Sternberg) Arno Schönberger ist wissenschaftlicher Mitarbeiter am Lehrstuhl für Öffentliches Recht (Prof. Dr. Reinhardt), beide Universität Trier. 
sensualen Werten verpflichtet sein, aber auch neue im laufenden politischen und juristischen Diskurs aufkommende Erscheinungen auffangen, diesen mithalten und sie angemessen abbilden zu müssen. In letzter Zeit - vor ziemlich genau einem Jahr - schlug vor allem die Diskussion um die Einführung der sogenannten "Ehe für alle" hohe Wellen, weil diese entweder über einen gewandelten Verfassungsbegriff in Bezug auf das Rechtsinstitut der Ehe nach Art. 6 Abs. 1 GG hergeleitet werden kann oder gegen eben jenen verstößt. Diese Omnipräsenz der schon seit jeher hoch im Kurs stehenden Verfassungsdiskussion bemerkte man dementsprechend natürlich auch in ganz besonderer Weise auf der diesjährigen Tagung. Sie befasste sich mit allen Aspekten einer Verfassung und nahm dabei auch vergangene und aktuelle Tendenzen von Verfassungskrisen in Deutschland, Europa und darüber hinaus in den Blick.

So näherte sich die Tagung dem Thema in sieben Panels mit jeweils unterschiedlichen Perspektiven, wobei die Referenten über einen Call of Papers ausgewählt wurden: 1. Verfassungsbeziehungen (rechtsgeschichtliche und rechtsphilosophische Grundlagen und Kontexte von Verfassungen: Verfassungswerdung - Eine sozialonthologische und sprechakttheoretische Analyse der Entstehung einer Verfassung und ihrer Akteure, Kathrin Strauß, Universität Münster; Verfassungspatriotismus, Eva Ricarda Lautsch, Universität Bochum), 2. Verfassungskräfte (Entfaltung durch Verfassungen selbst und Einwirkung auf Verfassungen: Verfassungsentwicklung als Diskurs zwischen Gesetzgebung, Vollziehung und Lehre, Dr. Josef Müllner, MMag, Universität Wien; Verfassungsnormativität im Recht der Politik, Sven Jürgensen, Universität Düsseldorf), 3. Verfassungsbilder (europa-, völkerrechtliche und nationalstaatliche Verfassungsrechtsinstitute und ihr Einfluss aufeinander: Vor- und Gegenbilder moderner Verfassungsurkunden, Annabelle Meier, Universität Würzburg; Gewaltenteilung und Gewaltengliederung in der transnationalen Konstellation, Dr. Johan Horst, LL.M. (Georgetown), Universität Bremen), 4. Verfassungssubjekte (Verfassungen als Abbild gesellschaftlicher Rahmenbedingungen und ihrer Akteure: Verfassungswandel messbar machen - Eine interdisziplinäre Betrachtung gesellschaftlicher und rechtlicher Entwicklungen homosexueller Paarbeziehungen, Franziska Spanner, M.A. und Simon Pschorr, beide Universität Konstanz; Verfassungssubjekte im Wandel - Kinderrechte ins Grundgesetz?, Isabel Lischewski, Universität Münster), 5. Digitalisierungsverfassung/ Verfassungsdigitalisierung (neuartige Herausforderungen für verfassungsrechtliche Strukturprinzipien: In Würde vor Ampel und Algorithmus - Verfassungsrecht im technologischen Wandel, Dr. Sebastian J. Golla, Universität Mainz; Die Rundfunkfreiheit: Ein Kind ihrer Zeit - aus der Zeit gefallen?, Friedrich Schmitt, Universität Freiburg), 6. Verfassungskrisen (Verfassung als Fluchtpunkt grundsätzlicher rechtlicher Probleme: Soziale Ungleichheit als Verfassungsherausforderung, Dr. Konstantin Chatziathanasiou, Universität Münster; Kann die Verfassung die Demokratie retten? - Verfas- 
sungsvergleichende Überlegungen zur Bedeutung wahlrechtlicher Regelungen für die Demokratie, Dr. Maria Bertel, Central European University Budapest; Das Bundesverfassungsgericht als "Hüter der Verfassung" - Zur Rolle und Bedeutung von Verfassungsgerichten in Krisenzeiten, Dr. Anne-Christin Gläß, LL.M. Eur, Universität Leipzig), 7. Verfassungswandel (evolutive Auslegungen und grundlegende Reformen sowie Änderungen des Verfassungstextes: Verfassungskontinuität durch Verfassungsänderung - Wie kann eine Verfassung ihre Dauerhaftigkeit sichern?, Dr. Matthias Lukan, LL.M. (WU), LL.B. (WU), Universität Wien; Die Schweizerische Bundesverfassung in beständigem Wandel - Spannungsfelder zwischen direkter Demokratie und Verfassung, Dario Picecchi, Universitäten Luzern/ Zürich; Änderung und Wandlung der Unionsverfassung seit dem Vertrag von Lissabon, Dr. Andreas Orator, LL.M. (NYU), Universitäten Heidelberg/ Wien).

Zurück aber zur eingangs erwähnten "Wiege der Demokratie": Der Eröffnungsabend zur Tagung fand direkt an einem Originalschauplatz deutscher Verfassungsgeschichte statt: Die Frankfurter Paulskirche, in der am 18. Mai 1848 die Deutsche Nationalversammlung zusammen trat und bis zum 30. Mai 1849 die Paulskirchenverfassung als erste gesamtdeutsche Verfassung erarbeitete, wurde an diesem Abend von der Frage getragen, was eine Verfassung nach damaligem und heutigem Verständnis im Wesen ausmacht. Dazu referierte mit Prof. Dr. Angelika Nußberger (Vizepräsidentin des Europäischen Gerichtshofs für Menschenrechte) eine eigens verfassungsrechtliche Akteurin. Ihre Festrede befasste sich damit, ob die Europäische Menschenrechtskonvention eine Verfassung für Europa darstellt. Ein ohne Frage spannendes Thema. Aber wäre es nicht zur Einordnung in den gesamten öffentlich-rechtlichen Diskurs eher interessant gewesen zu fragen, ob die in der Europäischen Menschenrechtskonvention niedergelegten Rechte und Mechanismen dazu geeignet sind, heutige Verfassungskrisen hinreichend bis verlässlich zu lösen? Auch wenn die Referentin letzten Endes auch zu einem großen Teil auf diese Frage eingegangen ist - insbesondere durch ihre inhaltlich-argumentativ überzeugende Auseinandersetzung mit der Deutung und Auslegung der Konventionsbestimmungen durch den Europäischen Gerichtshof für Menschenrechte als sogenanntes "living instrument" und damit auf der Grundlage aktueller und nicht vergangener sozialer und wirtschaftlicher Gegebenheiten - wirkte doch die eigentliche Vortragsformulierung, aus der Europäischen Menschenrechtskonvention als völkerrechtlichen Vertrag selbst einen klassischen Verfassungstext zu konstruieren, etwas gekünstelt bis gewollt.

Was insgesamt wohl der Natur der Sache und damit hier der enormen Breite des Verfassungsthemas geschuldet ist, kann dennoch nicht darüber hinwegtäuschen, dass so manches Thema in die Verfassungsecke gedrängt wurde und das eigentliche Anliegen der Tagung dadurch stellenweise etwas an Kontur verlor (gleichsam 
unter dem Stichwort "Alles ist Verfassung."). Zwar eignet sich gerade der Verfassungsbegriff wegen seiner Weite natürlich in sehr vielfältiger Hinsicht besonders gut zu dem stets auch erforderlichen übergeordneten Diskurs. Allerdings sind Verfassungen generell und insbesondere auch das deutsche Grundgesetz und darin die Grundrechte wegen der ihm und ihnen vom Bundesverfassungsgericht und der allgemeinen Dogmatik zugeschriebenen Funktion als objektive Wertordnung dazu geeignet, rechtliche Phänomene zu überformen und im Wege der verfassungskonformen Gesetzesauslegung nach ihrer eigenen Konsistenz aufzulösen. Denn letzten Endes lässt sich doch jedes rechtliche Problem eben immer auch in der Verfassungsdimension denken und fragen, ob und inwiefern eine soziale Fehlentwicklung als Verfassungsproblem, -herausforderung oder sogar -krise gedeutet werden kann. So dient außerdem umgekehrt jedes rechtliche Instrumentarium zur Lösung eines politisch-gesellschaftlichen Problems im weitesten Sinne auch der Wahrung verfassungsrechtlicher Werte. In dieser Herangehensweise stellte in seinem Grußwort am Eröffnungsabend der Tagung auch der Geschäftsführende Direktor des Instituts für Öffentliches Recht an der Universität Frankfurt Prof. Dr. Uwe Volkmann fest, dass sich über Verfassungen immer reden lässt. Dabei sollte man es aber gerade angesichts aktueller Entwicklungen nicht belassen und auf zunehmend populistische und autokratische Tendenzen auch und gerade innerhalb der Europäischen Union schauen. Sie darf nicht zur pauschalen Dauer- oder Notfallösung (oder wie Volkmann es sehr passend ausdrückt: zum reinen Bild oder zur bloßen Projektion) verkommen, mit der im Falle des Versagens anderer spezifischer Herangehensweisen rechtliche Probleme allein aufgrund der ihr zukommenden autoritativ-normativen Kraft gelöst werden. Denn andernfalls - und so drückt es auch Volkmann in seinem Grußwort aus - droht das Grundgesetz als unsere Verfassung auf lange Sicht zu verblassen.

Von diesem omnipräsenten aber im Ergebnis kleinen Detail abgesehen, war das Thema anlässlich der Verfassungsjubiläen überaus passend gewählt (wenn nicht angesichts der rein zeitlichen Nähe vielleicht sogar unvermeidbar) und in jeder Hinsicht sehr gut umgesetzt. Es wurde auf sämtliche Aspekte einer Verfassung eingegangen, von ihrer Entstehung, über ihre Abgrenzung zu anderen rechtlichen Erscheinungen, ihre einzelnen Bestandteile, die an ihrer Entstehung und Realisierung beteiligten Akteure sowie ihre immerwährenden Herausforderungen und schließlich auch ihre Fähigkeit, diesen trotzen und souverän antworten zu können, ohne dabei selbst unterzugehen. Der letzte Aspekt war es in ganz besonderer Weise, mit dem sich die Tagung in den aktuellen öffentlich-rechtlichen Diskurs einordnete und ihren Beitrag hierzu lieferte. 


\section{Tagungsprogramm}

Mit dem Präsidenten des Bundesverfassungsgerichts Herrn Prof. Dr. Dr. h.c. Andreas Voßkuble konnte das Frankfurter Team auch für das Abendprogramm des zweiten Veranstaltungstages einen zum Tagungsthema prädestinierten Festredner gewinnen. Der Karlsruher Verfassungsrichter referierte unter dem Titel "Karlsruhe Unlimited? - Zu den Grenzen der Verfassungsgerichtsbarkeit" über die Kritik, die an dem ausgeprägten Einfluss des Bundesverfassungsgerichts auf grundlegende politische Entscheidungen in Deutschland und Europa aufgrund seiner weitreichenden Kompetenzen vorgetragen wird. Er hielt dem jedoch entgegen, dass auch in der Bundesverfassungsgerichtsbarkeit, nicht zuletzt durch die Organisation als Kollegialorgan und die Etablierung des Sondervotums effektive Selbstkontrollmechanismen angelegt seien. Das Format des Festvortrags hat dabei dieses Jahr insoweit eine programmatische Neuerung erfahren, dass zwei Kollegen die Gelegenheit geboten wurde, den Festvortrag eigens zu kommentieren. Aus zahlreichen Bewerbungen im Rahmen eines Calls for Commentators wurden Quirin Weinzierl, LL.M. (Yale) (Deutsches Forschungsinstitut für Öffentliche Verwaltung, Speyer) und Dr. Andrej Lang (Martin-Luther-Universität Halle-Wittenberg) ausgewählt, die in ihren Repliken den Ausführungen Voßkubles respektvoll und souverän entgegentraten. Dass diese Modifikation des klassischen Festvortrags bei den Tagungsteilnehmern Anklang fand, zeigte sich nicht nur in den zahlreichen Bewerbungen bei dem Call of Commentators, sondern auch in der positiven Resonanz im Rahmen der Aussprache am Ende der Tagung. Es wäre daher erstrebenswert, dieses Format auch bei künftigen Assistententagungen zu wiederholen, wobei kein Zwang bestehen sollte, stets einen kommentierten Festvortrag zu implementieren, sondern je nach Tagungsthema auch an den etablierten Kategorien festzuhalten.

Als weitere Neuerung trug 2019 mit der Soziologin Franziska Spanner erstmals in der Geschichte der öffentlich-rechtlichen Assistententagung eine fachfremde Referentin vor. Am Beispiel homosexueller Paarbeziehungen referierte die Konstanzer Doktorandin gemeinsam mit Simon Pschorr über die diesbezügliche gesellschaftliche, aber auch rechtliche Entwicklung. Sie legten in aufeinander aufbauenden und sich als Referenten und ihre Fachrichtungen gegenseitig ergänzenden Teilaspekten ihres Beitrags dar, inwiefern auch das Bundesverfassungsgericht auf die gesellschaftliche Entwicklung reagierte und trotz gleichlautendem Verfassungsrecht die rechtliche Stellung homosexueller Paarbeziehungen in unterschiedlichen Jahrzehnten verfassungsrechtlich unterschiedlich einordnete. Die Vortragenden thematisierten dabei auch, inwiefern sich fundamentale Änderungen der Verfassungsauslegung bei gewandelten gesellschaftlichen Wertvorstellungen rechtfertigen lassen. In der sich anschließenden Diskussion wurde heftig aber stets sachlich darüber gestritten, ob es sich mit der Funktion der Verfassung vereinbaren lässt, wenn die 
Verfassungsauslegung gewandelten Wertvorstellungen folgt, oder ob nicht die Verfassung ihrerseits zwingende Wertvorstellungen vorgeben müsse.

Für eine juristische Fachtagung untypisch war auf den ersten Blick der Vortrag von Kathrin Stauß über die Entstehung von Verfassungen. Sie versuchte, den Ursprung gesellschaftlicher Regeln zu eruieren und bediente sich dazu auch sprachtheoretischer und sozialonthologischer Methoden. Ihr Ansatz war sicher für die meisten Zuhörer Neuland und schon durch die Verwendung zahlreicher ungewohnter Fachbegriffe war es teilweise schwer, die Analysen der Vortragenden zu erfassen. Nichtsdestotrotz ist der Versuch, Gegebenheiten, die für viele Juristen einfach so als gegeben hingenommen werden, mit der Sicht fachfremder Methoden zu hinterfragen und zu analysieren, durchaus begrüßenswert. Hierdurch nämlich wird die eigene Sicht geschärft und der Blick auf Aspekte gerichtet, die bislang nur geringe Aufmerksamkeit erfahren haben.

In der Tagungsaussprache nach Beendigung des eigentlichen Programms kam von einzelnen Teilnehmern der Wunsch auf, künftig mehr fachfremden Referenten die Möglichkeit zu geben, auf der Assistententagung vorzutragen, um somit die Diskussionsgrundlage breiter aufzubauen. Dem wurde aber auch entgegengehalten, dass das ohnehin begrenzte Angebot, für junge Wissenschaftler im Öffentlichen Recht vor einem vergleichsweise großen Fachpublikum vorzutragen, nicht weiter eingeengt werden solle. Beide Ansichten mögen ihre Berechtigung haben. Anzumerken bleibt bei dieser Gelegenheit jedoch einzig, dass gerade die Form des kombinierten Vortrags, bei dem stets ein juristischer und ein nicht-juristischer Experte zusammen referieren, ein interessantes und durchaus zukunftsträchtiges Format bietet, bei dem ferner eine Beschneidung der Präsentationsmöglichkeit für die Assistentenkollegen nicht zu befürchten sein dürfte.

\section{Fazit}

Alles in allem war die diesjährige Tagung eine durch und durch runde und gelungene Veranstaltung, die sich gleichermaßen der Vergangenheit wie der Zukunft der Verfassungsdiskussion als Bestandteil der gesamt-öffentlich-rechtlichen Debattenkultur bewusst war und stetig vor Augen führte. Zur Zukunft derselben gehören in ganz besonderer Weise auch das Thema der Digitalisierung und die Herausforderungen, vor die sie die Begriffe nationaler und internationaler Rechts- und Verfassungsordnung stellt. All diese Fragen werden Gegenstand der nächsten Tagung welche dann bereits ihr 60-jähriges Jubiläum feiert - unter dem Arbeitstitel "Der digitalisierte Staat" sein. 